\title{
Probing the Origin of in situ Generated Nanoparticles as Sustainable Oxidation Catalysts
}

\author{
Christopher S. Hinde $^{a}$, Sivan Van Aswegen ${ }^{a}$, Gillian Collins ${ }^{\text {bc }}$, Justin D. Holmes ${ }^{\text {bc }}$, T. S. Andy Hor ${ }^{\text {d }}$ and \\ Robert Raja*a
}

${ }_{5}$ Received (in $\left.X X X, X X X\right)$ Xth $X X X X X X X X X 20 X X$, Accepted Xth XXXXXXXXX 20XX

DOI: 10.1039/b000000x

A novel method for the in situ generation of catalytically active small metal nanoparticles, by anion extrusion on a parent porous copper chloropyrophosphate framework, have

10 been developed to generate gold, platinum and palladium nanoparticles for sustainable catalytic oxidations using molecular oxygen as the oxidant. Transmission electron microscopy coupled with detailed structural and physicochemical characterisation, in combination with in-depth 15 kinetic analysis have afforded profound insights into the nature of the active site for facilitating structure-property correlations.

\section{Introduction}

The selective oxidation of hydrocarbons and alcohols plays a 20 decisive role in the synthesis of vital ingredients for the finechemical and pharmaceutical industries. Current industrial processes involve the use of stoichiometric quantities of inorganic oxidants, largely homogeneous chromium(VI) based materials, ${ }^{1}$ that result in the generation of excessive quantities of waste, 25 notwithstanding the debilitating impacts on the environment through use of caustic reagents and non-biodegradable solvents. From the point of view of sustainable chemistry, this sector of the chemical industry is faced with the particular challenge to utilise benign oxidants, such as molecular oxygen, to eliminate these 30 toxic waste products and expensive reagents, whilst simultaneously striving to achieve high degrees of activity and selectivity, through the deployment of heterogeneous catalysts. Utilising dry air, as the source of active oxygen, presents an attractive alternative, due to its natural abundance, low-costs ${ }^{2}$, 35 mild reaction conditions and better tunability for minimising the generation of by-products. However, significant challenges still remain in the quest for the discovery and design of single-site heterogeneous catalysts that can effectively activate molecular oxygen, under mild conditions, affording high catalytic turnovers 40 whilst maintaining the high selectivities of their homogeneous counterparts.

The selective oxidation of benzyl alcohol to benzaldehyde ${ }^{3}$ is of paramount importance; due to its use as a pharmaceutical intermediate, as well as being an important precursor for the 45 generation of plastic additives, photographic chemicals and dyes. ${ }^{4}$ Potential industrial methods afford inferior selectivities towards the target aldehyde; with over-oxidation (as a result of the

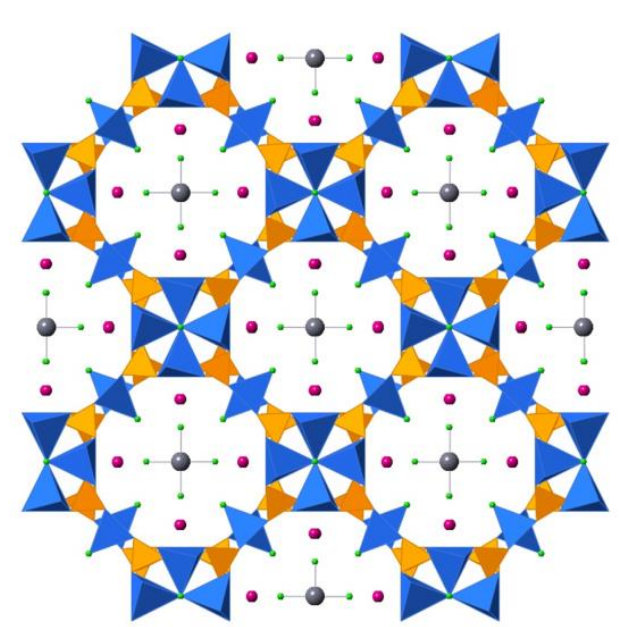

Fig. 1 Crystal structure of the $\mathrm{PtCl}_{4}{ }^{2-}$ supported $\mathrm{Rb}_{9} \mathrm{Cu}_{6}\left(\mathrm{P}_{2} \mathrm{O}_{7}\right)_{4} \mathrm{Cl}_{2}$ 50 framework ( $\mathrm{Pt}$ - grey spheres, $\mathrm{Cl}$ - green spheres, $\mathrm{Rb}$ - pink spheres, $\mathrm{Cu}$ - blue polyhedral, $\mathrm{P}$ - orange polyhedral, oxygen omitted for clarity)

demanding reaction conditions employed) to benzoic acid, and esterification to benzyl benzoate being the main impediments hindering successful commercial implementation. ${ }^{5}$ The use of 55 more environmentally-benign oxidants, such as hydrogen peroxide $\left(\mathrm{H}_{2} \mathrm{O}_{2}\right)$ (where water is the main by-product) with heterogeneous catalysts, ${ }^{5,6}$ have not proved effective due to the high costs associated with the production of $\mathrm{H}_{2} \mathrm{O}_{2}$ and concomitant low selectivities for the target aldehyde.

60 Nanoparticle catalysts have shown significant promise in a variety of chemical transformations; but more specifically in aerobic oxidations, with reports of $\mathrm{Pt}$ and $\mathrm{Pd}$ supported nanoparticles alloyed with $\mathrm{Au}$ displaying impressive conversions. ${ }^{7}$ Recent impressive studies even show the potential 65 of alloyed nanoparticles for catalysing more demanding activation of primary $\mathrm{C}-\mathrm{H}$ bonds in toluene, ${ }^{8}$ as well as in the selective synthesis of hydrogen peroxide, ${ }^{9}$ which pave the way for overcoming some of the earlier limitations. ${ }^{5,6}$ Heterogeneous nanoparticle catalysts have mostly been supported on porous 70 carbon ${ }^{10}$ or transition-metal oxides, ${ }^{11}$ with synthetic techniques based around depositing nanoparticles on a pre-prepared material surface. More recently zeolites, ${ }^{12}$ metal-organic frameworks $(\mathrm{MOFs})^{13}$ and other porous silica-based materials ${ }^{14}$ have been explored as potential hosts for functionalising nanoparticle 75 catalysts. Common synthetic methodologies include wet- 

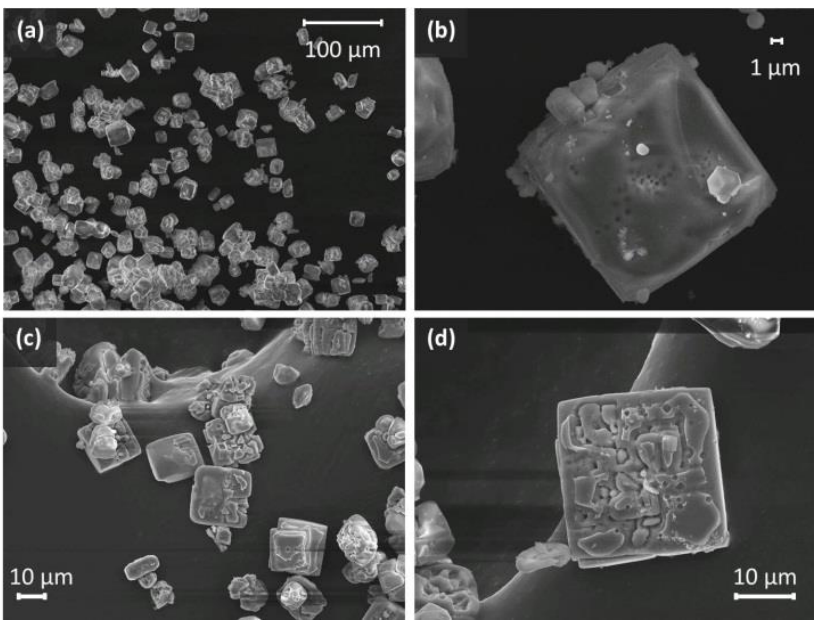

Fig. 2 SEM Micrographs of the as-synthesised Pt catalyst (a and b) and Pd catalyst ( $c$ and d) showing homogeneous cubic crystal morphology and single-crystals respectively
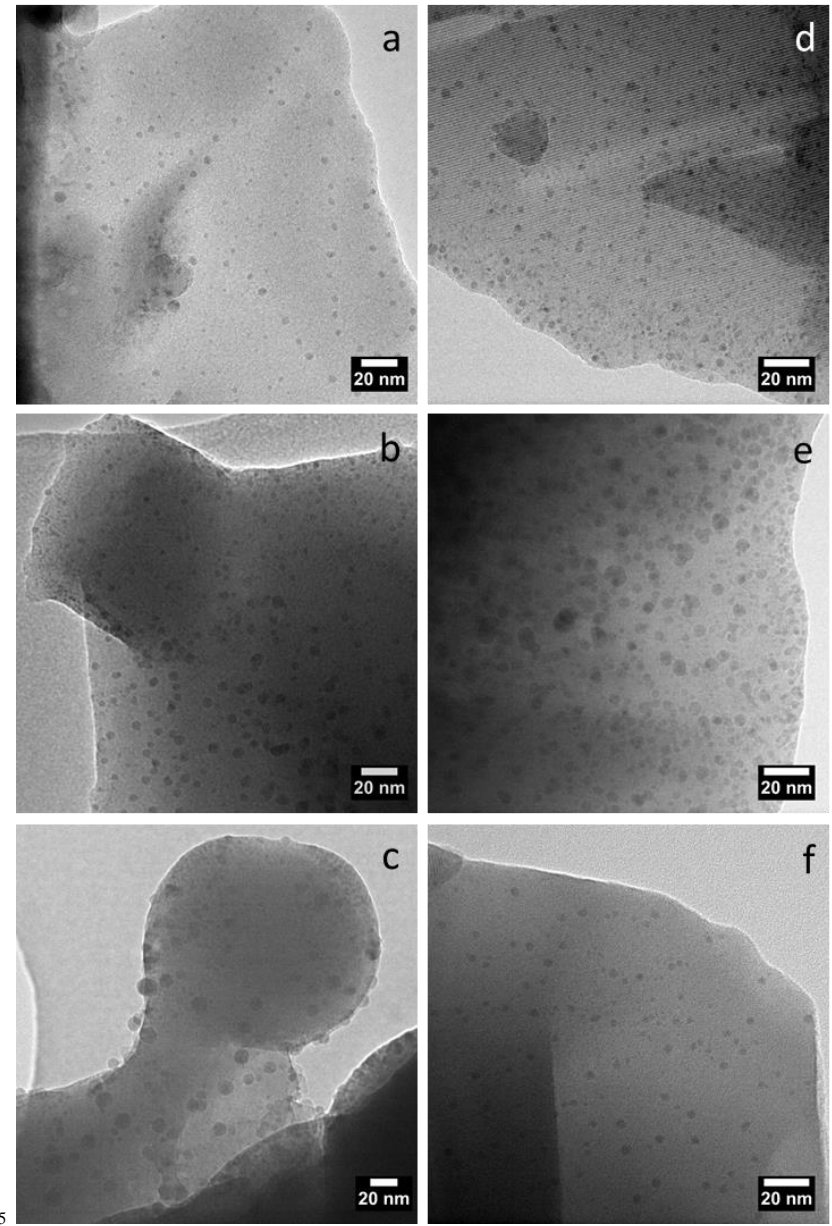

Fig. 3 TEM images of (a) Au (b) Pt and (c) Pd catalysts after calcination. (d) Au (e) Pt and (f) Pd catalysts after the oxidation reaction.

impregnation, ${ }^{15}$ deposition-precipitation ${ }^{16}$ and polymer-stabilised colloidal deposition; ${ }^{17}$ where precise control of nanoparticle size 10 and distribution have been achieved employing polyvinylalcohol (PVA) and polyvinylpyrrolidone (PVP) as polymer-stabilising agents. Catalytic studies for $\mathrm{Au}$ nanoparticles have revealed that the activity is inversely proportional to the particle size, especially below $10 \mathrm{~nm},{ }^{18}$ thus highlighting the importance of
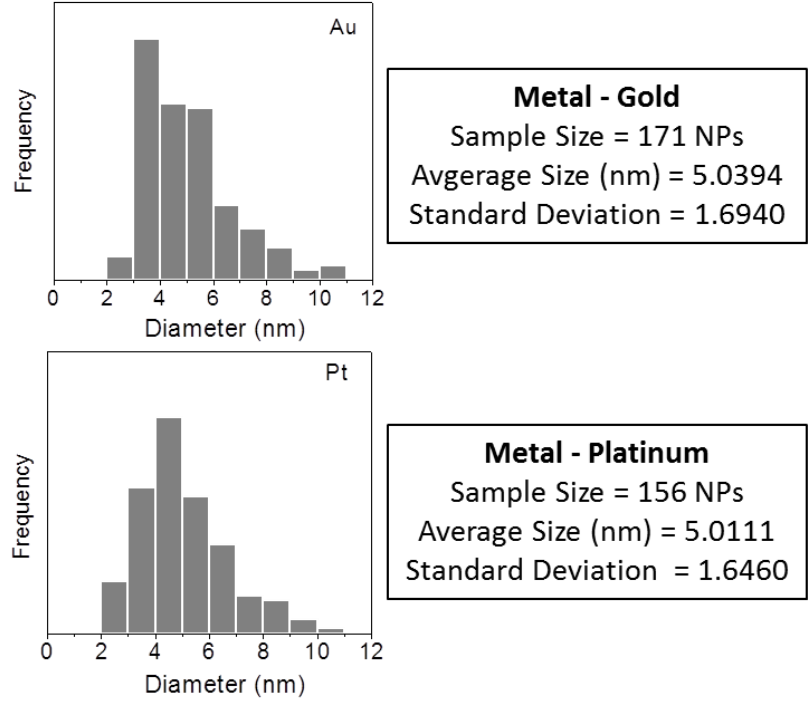

Metal - Platinum

Sample Size $=156$ NPs

Average Size $(\mathrm{nm})=5.0111$

Standard Deviation $=1.6460$

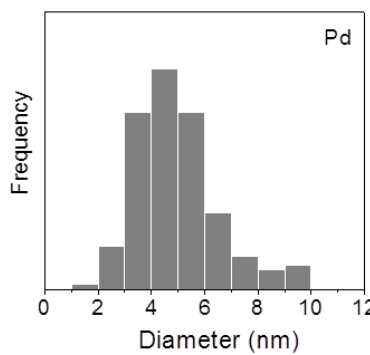

\section{Metal - Palladium}

Sample Size $=162$ NPs

Avgerage size $(\mathrm{nm})=4.9168$

Standard Deviation $=1.5844$

Fig. 4 Histogram showing the particle size distribution for the Au, Pt and Pd catalysts

size and shape control in the synthesis. Furthermore, the use of noble metal nanoparticles in the oxidation of benzyl alcohol 20 alludes to favourable interactions between the gold surface and the $\pi$-system of the aromatic ring to enhance the overall efficiency of the active site. ${ }^{19}$

Very recently, a synthetic method for the generation of $\mathrm{Au}$ particles, via an extrusion method from an $\left[\mathrm{AuCl}_{4}\right]^{-}$supported 25 copper chloropyrophosphate with an analogous framework as CU-2 was reported. ${ }^{20,21}$ Herein, we adopt a modified approach for the generation of $\mathrm{Pt}$ (Fig. 1) and Pd nanoparticles for the aerobic selective oxidation of benzyl alcohol. Using a combination of electron microscopy, coupled with a detailed 30 kinetic analysis of the reaction profile, has enabled structureproperty correlations to be established with its Au-based counterpart.

\section{Experimental Section}

Chemicals for synthesis and catalytic tests were purchased from ${ }_{35}$ Sigma Aldrich, Fisher Scientific or Acros Organics and used without further purification. X-Ray diffraction (XRD) patterns were collected either on a Bruker $\mathrm{C} 2$ general area detector diffraction system (GADDS) or a Bruker D2 Phaser diffractometer. SEM images and EDX analysis were collected 40 with a JEOL JSM-6500F field emission gun microscope. TEM analysis was carried out on a JEOL 2100 electron microscope at an operating voltage of $200 \mathrm{kV}$.

For the sake of consistency, an analogous synthetic approach was adopted for the catalysts evaluated in this study. Copper(II) 
fluoride (0.1168 g, $1.150 \mathrm{mmol}), 85$ w.t.\% orthophosphoric acid

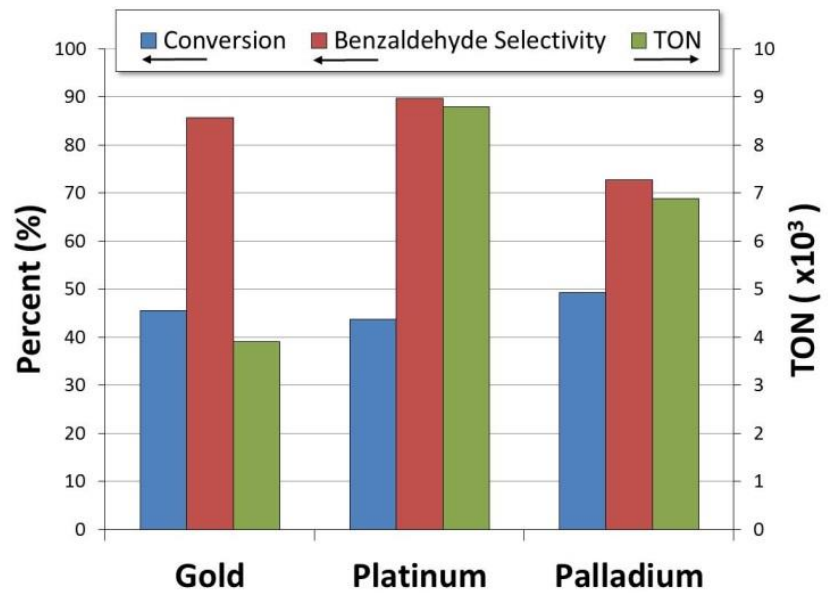

Fig. 5 Conversion and selectivity* (left) for the aerobic oxidation of benzyl alcohol (after $4 \mathrm{~h}$ ) using the three catalysts employed in this study 5 along with their associated turnover numbers (TON) shown on the right. Note: * remainder benzoic acid.

Key: blue- conversion, red- selectivity, green- TON

Reaction conditions: benzyl alcohol $(1.00 \mathrm{~g})$, catalyst $(50 \mathrm{mg})$, diglyme internal standard $(0.85 \mathrm{~g})$, tert-butanol $(30 \mathrm{~mL}), \mathrm{T}=130^{\circ} \mathrm{C}$, Air (oxidant) 10 $\cong 2 \mathrm{MPa}$

(0.2 mL, $2.922 \mathrm{mmol}), 50$ w.t.\% RbOH (0.28 mL, $2.382 \mathrm{mmol})$ and a source of $\mathrm{MCl}_{\mathrm{x}}$; $\mathrm{HAuCl} 4(0.0978 \mathrm{~g}, 0.288 \mathrm{mmol}), \mathrm{K}_{2} \mathrm{PtCl}_{4}$ $(0.1196 \mathrm{~g}, 0.288 \mathrm{mmol})$ or $\mathrm{K}_{2} \mathrm{PdCl}_{4}(0.0940 \mathrm{~g}, 0.288 \mathrm{mmol})$ were ixed in the Teflon ${ }^{\circledR}$ liner of a custom-made $23 \mathrm{~mL}$ hydrothermal 15 vessel. The vessel was sealed and heated to $448 \mathrm{~K}$ for 2 days.

Products formed as brilliant green cuboid crystals for both the $\mathrm{Au}$ and Pt material, and as light brown crystals for the Pd material. All materials were calcined in air at $773 \mathrm{~K}$ for 16 hours to generate the active nanoparticle catalysts. After calcination, the ${ }_{20} \mathrm{Au}$ and $\mathrm{Pd}$ materials appeared unchanged in colour; but the Pt catalyst changed to a darker khaki-green.

Catalytic reactions were carried out in a $75 \mathrm{~mL}$ PTFE lined, stainless-steel, high-pressure batch reactor. The reactor was charged with benzyl alcohol (1 g), diethylene glycol dimethyl 25 ether $(0.85 \mathrm{~g})$ as an internal standard, tert-butanol $(30 \mathrm{~mL})$ as a solvent and catalyst $(50 \mathrm{mg})$. The reactor was pressurised with $\cong 20$ bar of dry air, stirred and heated for the required amount of time. Small aliquots of the reaction mixture were taken carefully periodically for GC analysis. Samples were analysed by GC 30 (PerkinElmer, Clarus 480) using an Elite-5 column equipped with a flame ionisation detector (FID). Products were identified against authenticated standards and quantified by calibration to obtain response factors (RF) against the known internal standard.

\section{Results and Discussion}

35 To effectively characterise each catalyst, powder XRD, SEM and TEM analyses (See ESI for complete data sets) were performed and evaluated to confirm the structure of the parent material and to determine the presence of metallic nanoparticles, where necessary.

40 SEM analyses have been previously used to study the morphology of the copper chloropyrophosphate crystals with tetrachloroaurate anions supported in the 1D pores. ${ }^{21}$ Employing similar imaging methodologies, the morphology of the platinum chloride and palladium chloride supported phases have been 45 shown to have a homogenous distribution of cubic crystals across

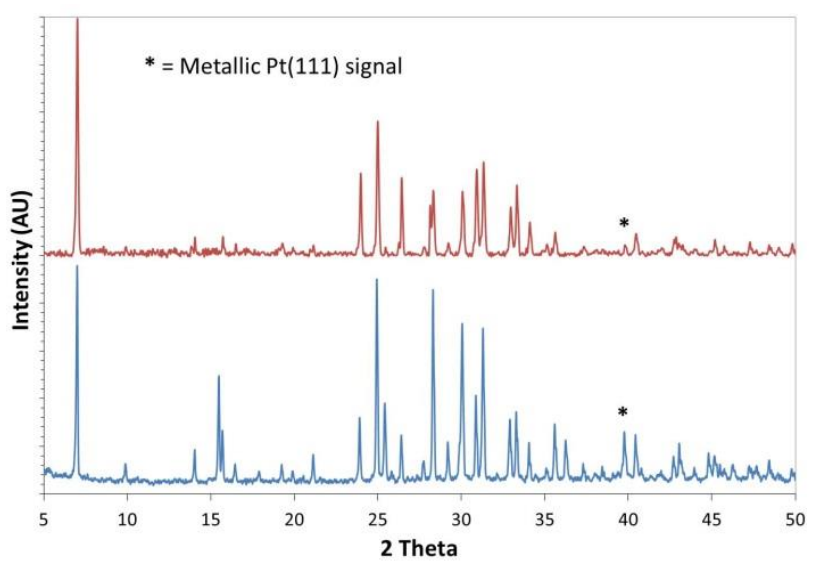

Fig. 6 Powder XRD patterns of the calcined platinum catalyst (blue line) and the catalyst post-catalysis (red line). Note: the used catalyst has not been further calcined in this case.

50 the synthesised material (Fig. 2). Higher magnification images of the single-crystals have revealed that the surfaces on the $\mathrm{Pd}$ analogue appear roughened with clear features; whereas the $\mathrm{Pt}$ crystal faces are relatively smooth. It is possible that elevated Pd, or even chlorine content, in the synthesis procedure could 55 influence the growth of crystals in the hydrothermal process.

Powder XRD patterns of the calcined materials (ESI Fig. 1.11.2) clearly show signals that can be attributed to the elemental metal crystalline phase, which are absent on the simulated pattern and the blank framework diffractogram. Moreover, these particles ${ }_{60}$ formed by extrusion are conspicuous by their absence on the surface of the as-synthesised samples. The SEM images along with their associated diffraction patterns confirm the phase-purity and homogeneous crystal morphology observed for each material; further highlighting the ease and reproducibility of the 65 synthetic procedure.

TEM analysis of the calcined $\mathrm{Au}, \mathrm{Pt}$ and Pd catalysts (Fig. 3 ac) reveal the presence of well-defined, small metallic nanoparticles that are uniformly distributed throughout the chloropyrophosphate framework. The majority of the 70 nanoparticles display a spherical morphology with diameters ranging from 2-10 nm. Detailed size distribution histograms (Fig. 4) give a perspective on the particle sizes; whilst the average particle sizes are similar, it is noted that the distribution of gold nanoparticles shows the most asymmetry with modal preference 75 of smaller particles between 3-4 $\mathrm{nm}$. It was interesting to note that the TEM analysis also identified the presence of larger faceted nanocrystals (ESI Fig. 7.1) in the Pd-catalyst. Structural characterisation of the catalysts after the oxidation reaction (postcatalysis, Fig. 3 d-f) demonstrate that the metal nanoparticles 80 remain intact and are fairly well-dispersed on the support, which augurs well for their recyclability (see also Fig. 6).

We focussed our attention on evaluating the catalytic properties of each of the $\mathrm{Au}, \mathrm{Pt}$ and $\mathrm{Pd}$ catalysts in order to decipher the merits of the individual metal constituents and their 85 implicit role in the overall activity (TON) and selectivity in the aerobic oxidation reaction. It was noted that, under comparable conditions, the Pd analogue was slightly more active (in terms of 
overall conversion only) than its corresponding Au or Pt-based counterparts; albeit displaying a lower selectivity for the desired product (benzaldehyde) (Fig. 5). In order to get a more accurate
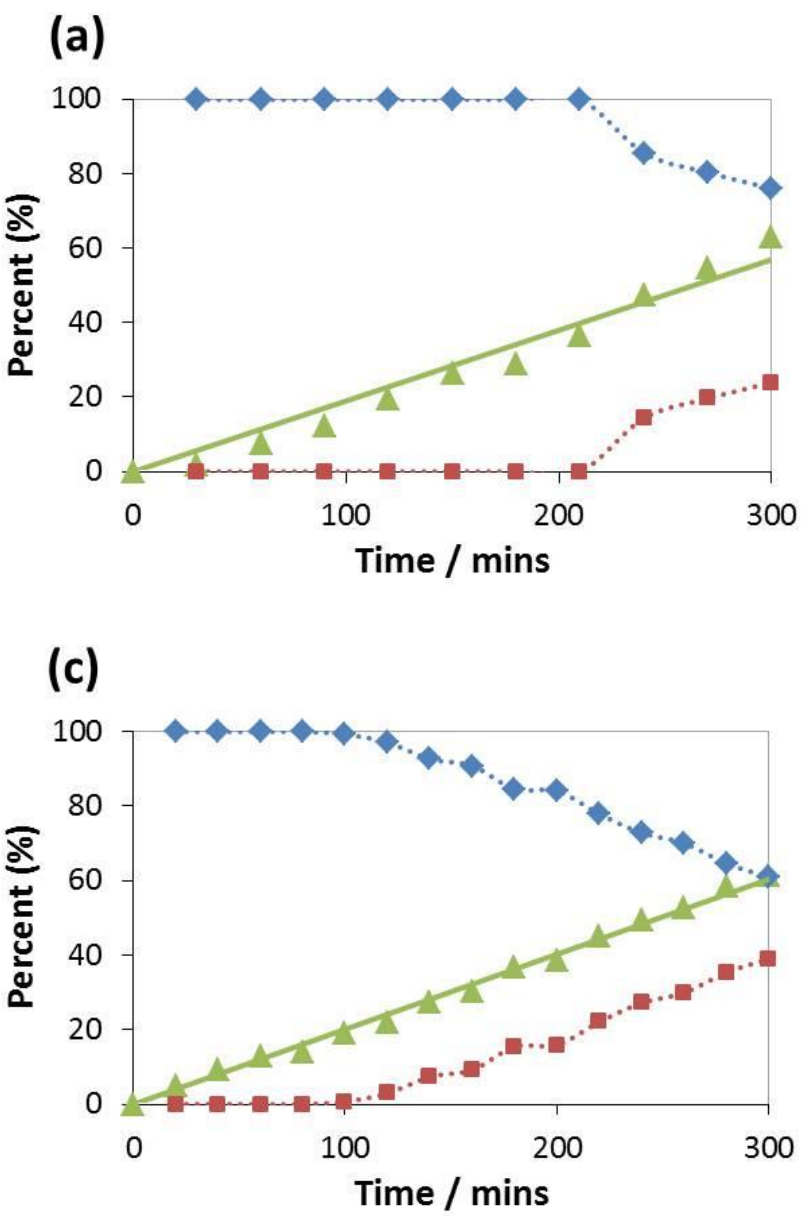

Fig. 7 Kinetic plots for the aerobic oxidation of benzyl alcohol using gold (a), platinum (b) and palladium (c) nanoparticle catalysts.

Key: Conversion (green triangles), benzaldehyde selectivity (blue diamonds) and benzoic acid selectivity (red squares). See Figure 5 for 10 reaction conditions.

interpretation of the intrinsic reactivity of these catalysts, we have normalised the overall activity per mole of metal present in each catalyst (using ICP measurements to accurately determine the metal loadings (see ESI Tables 4.2-4.4)) and turnover numbers 15 (TON) have been calculated (see Fig. 5 right). These findings were highly revealing: despite the fact that an analogous synthetic approach was adopted for preparing all three catalysts, the Ptbased catalyst displays impressive TON $(8,789)$ when compared to its $\mathrm{Pd}(6,882)$ and Au-based counterparts $(3,911)$. It is highly

20 likely that site-isolation plays an important role in facilitating the higher catalytic TON with the former catalyst, which is consistent with our observations seen earlier in the SEM and TEM micrographs (Fig. 2 and 3). Further structural characterisation is currently underway to probe the origin these isolated active sites 25 and their implicit role in the catalytic process. It is also noteworthy that larger faceted nanocrystals were detected with the Pd-based catalyst, which could well be responsible for the lower TON and selectivity associated with this catalyst.

It is well known ${ }^{22}$ that nanoparticle size and degree of site-
30 isolation are crucial factors that lead to the generation of singlesites that are responsible for boosting the overall catalytic efficiency in nanoparticle-based catalysts that were previously

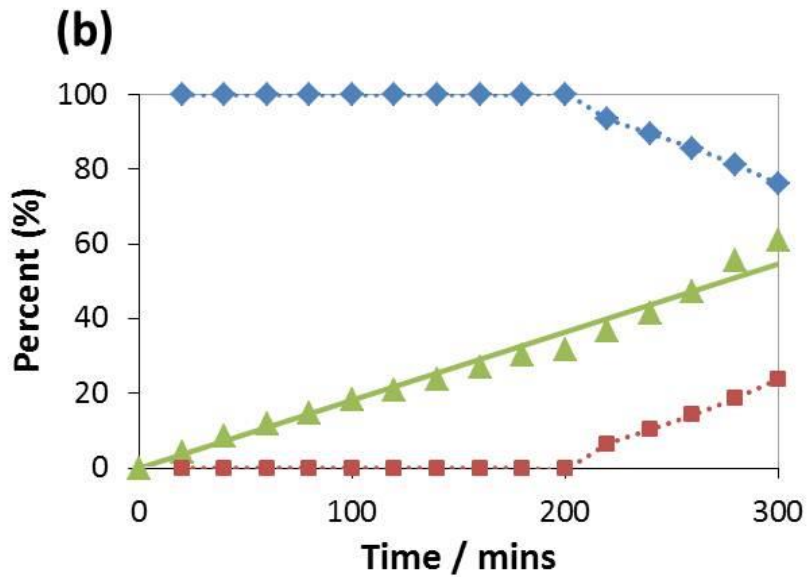

35 studied $^{23}$ for catalytic oxidations and hydrogenations. ${ }^{24}$ Whilst these studies focussed on using cluster-based precursors for generating nanoparticles (by post-synthesis deposition or grafting on mesoporous supports), this novel approach illustrates a "onepot" in situ method for achieving isolated active sites in porous 40 chloropyrophosphate-based framework architectures; where the inherent nature of the framework can be advantageously tailored for efficient shape-selective catalytic oxidations using molecular oxygen.

Stringent recycle tests show that these materials can be ${ }_{45}$ ffectively reused as heterogeneous catalysts for a minimum of three cycles, with little to no drop in performance or selectivity.

ICP analysis (ESI Table 4.1) confirms no leaching of metal species into the reaction solution and therefore proves that the catalyst is acting in a "true" heterogeneous fashion. The structural 50 integrity of these materials was analysed, post catalysis, by comparing powder XRD patterns before and after reaction cycles (Fig. 6 and ESI Fig. 2.1-2.2). It was noteworthy that, the parent framework resists degradation after exposure to the reaction conditions (temperatures, pressures, solvent, etc.), retaining much 55 of its characteristic phase-purity and structural integrity. It was also reassuring that the TEM analysis, post catalysis (Fig. 3 (d) (e)) reveals the presence of well-defined and well-distributed nanoparticles with no visible indications of aggregation or agglomeration, that could lead to potential sintering and 60 deactivation of the catalyst.

Detailed kinetic studies were carried out for each of the $\mathrm{Au}, \mathrm{Pt}$ and Pd catalysts (Fig. 7), with catalytic profiles being constructed for the consumption of benzyl alcohol with respect to time at varying temperatures between 373-403 K (see also ESI Fig. 3.165 3.6). It was ascertained from these studies that the rate is zero order with respect to the concentration of the substrate for all the analogues.

The calculated activation energies are in good agreement with those found in the literature relating to nanoparticle catalysts; ${ }^{7 \mathrm{c}}$ 70 whilst proving to be lower than those reported for the homogenous nitric acid oxidation of benzyl alcohol ${ }^{25}$ and other heterogeneous catalysts based on molybdenum and tungsten. ${ }^{6 \mathrm{~b}}$ 
Interestingly for the Pd-based catalyst, as noted from the kinetic plot (Fig. 7(c)), generation of benzoic acid occurs after just 90 minutes of reaction at $403 \mathrm{~K}$; whereas the $\mathrm{Pt}$ and $\mathrm{Au}$ catalysts afford very high selectivities towards the desired benzaldehyde 5 up to 200 minutes (at comparable levels of conversion). To further explore this phenomena, analogous reactions carried out at lower temperatures $(393 \mathrm{~K})$ show that the Pt catalyst maintains exclusive selectivity for the aldehyde for the entire duration of the reaction $(5 \mathrm{~h})$, while the $\mathrm{Pd}$ catalyst leads to the production of 10 benzoic acid after 200 mins (ESI Fig. 3.7). From these preliminary catalytic findings, it is particularly evident that the kinetic plots and TONs (Fig. 5 and 7) reveal that the Pt-based material is the most active and selective of the three catalysts for the aerobic oxidation of benzyl alcohol. With further refinements 15 to the synthetic protocol, by way of optimising the calcination temperature and/or altering the degree of hydrophobicity, adequate scope exists for tuning and controlling the size of the nanoparticles and its degree of site-isolation for attaining improved catalytic rates and turnovers, that would lead to the 20 development of stable, versatile catalysts for industrially significant selective oxidations using molecular oxygen.

\section{Prospects and Potential}

The development of a novel design strategy for the "one-pot" in 25 situ generation of metallic nanoparticle active sites, that could be isolated and heterogenised within a porous copper chloropyrophosphate framework, has resulted in the generation of highly active and selective catalysts for the activation of molecular oxygen. The size, shape and morphology of the 30 individual nanoparticles ( $\mathrm{Au}, \mathrm{Pt}$ or $\mathrm{Pd}$ ) that could be effectively confined within these novel anion-exchange host frameworks, opens up a new avenue of research for creating well-dispersed, single-sites that are stable and versatile in industrially-significant, selective catalytic oxidations, using benign oxidants such as air. 35 High catalytic turnovers coupled with superior activation energies, compared to other homogeneous and analogous heterogeneous systems, offers considerable scope for the deployment of heterogeneous, metal chloride precursor materials, for advantageously boosting the activity and selectivity in target 40 catalytic reactions. Whilst there is immense potential for the designed porous framework host to be catalytically active in itself; metal nanoparticle-framework interactions and metal-metal interactions (e.g. multimetallic nanoparticles) can be suitably engineered and investigated for eliciting synergistic 45 enhancements $^{6-8,}$ 21-23 in a range of sustainable catalytic transformations. From a design perspective, this route also offers a broader, complementary scope for alloying a range of metals and oxophiles that have hitherto been unexplored or have yet remained elusive using traditional synthetic methodologies.

\section{${ }_{50}$ Acknowledgements}

We would like to thank EPSRC (UK), and A*STAR, Singapore for funding. $\mathrm{CSH}$ thanks the University of Southampton for a VC scholarship. JDH acknowledges financial support from Science Foundation Ireland (Grant: 08/CE/I1432).

\section{${ }_{55}$ Notes and references}

${ }^{a}$ Faculty of Natural and Environmental Science (Chemistry), University of Southampton, Southampton, UK, SO17 1BJ. Tel: (+44)23 8059 2144; E-mail:R.Raja@soton.ac.uk

${ }^{b}$ Department of Chemistry and Tyndall National Institute, University ${ }_{60}$ College Cork, Cork, Ireland.

${ }^{c}$ Centre for Research on Adaptive Nanostructures and Nanodevices (CRANN), Trinity College Dublin, Dublin 2, Ireland

${ }^{d}$ Institute of Materials Research and Engineering (IMRE), A*STAR, 3, Research Link, Singapore 117602

${ }_{65} \dagger$ Electronic Supplementary Information (ESI) available: Full catalytic studies are presented with kinetic and arrhenius plots of all materials. SEM micrographs for each as-synthesised and calcined material with associated EDX data are provided, TEM micrographs for selected samples, powder XRD patterns and ICP data are also included. See 70 DOI: $10.1039 / \mathrm{b} 000000 \mathrm{x} /$

1 J.-E. Backvall, Modern Oxidation Methods, Wiley-VCH, Weinheim, 2005.

2 R. A. Sheldon, Chem. Soc. Rev., 2012, 41, 1437.

753 H. W. Chen, A. Murugadoss, T. S. A. Hor and H. Sakurai, Molecules, 2011, 16, 149.

4 R. A. Sheldon and H. Van Bekkum, Fine Chemicals through Heterogeneous Catalysis, Wiley-VCH, Weinheim, 2001.

5 V. R. Choudhary, D. K. Dumbre and S. K. Bhargava, Ind. Eng. $80 \quad$ Chem. Res., 2009, 48, 9471; G. Ming-Lin and L. Hui-Zhen, Green Chemistry, 2007, 9, 421.

6 US Pat., 5473 101, 1995; M. P. Chaudhari and S. B. Sawant, Chem. Eng. J., 2005, 106, 111.

7 J. Pritchard, L. Kesavan, M. Piccinini, Q. He, R. Tiruvalam, N. 85 Dimitratos, J. A. Lopez-Sanchez, A. F. Carley, J. K. Edwards, C. J. Kiely and G. J. Hutchings, Langmuir, 2010, 26, 16568; A. M. Henning, J. Watt, P. J. Miedziak, S. Cheong, M. Santonastaso, M Song, Y. Takeda, A. I. Kirkland, S. H. Taylor and R. D. Tilley, Angew. Chem. Int. Ed., 2013, 52, 1477; D. I. Enache, J. K. Edwards, 90 P. Landon, B. Solsona-Espriu, A. F. Carley, A. A. Herzing, M. Watanabe, C. J. Kiely, D. W. Knight and G. J. Hutchings, Science, 2006, 311, 362 .

8 L. Kesavan, R. Tiruvalam, M. H. A. Rahim, M. I. bin Saiman, D. I. Enache, R. L. Jenkins, N. Dimitratos, J. A. Lopez-Sanchez, S. H.

95 Taylor, D. W. Knight, C. J. Kiely and G. J. Hutchings, Science, 2011, 331, 195.

9 J. K. Edwards, B. Solsona, E. N. N, A. F. Carley, A. A. Herzing, C. J. Kiely and G. J. Hutchings, Science, 2009, 323, 1037.

10 D. Tasis, N. Tagmatarchis, A. Bianco and M. Prato, Chem. Rev., 100 2006, 106, 1105; M. Endo, Y. A. Kim, M. Ezaka, K. Osada, T. Yanagisawa, T. Hayashi, M. Terrones and M. S. Dresselhaus, Nano Lett., 2003, 3, 723 .

11 N. Zheng and G. D. Stucky, J. Am. Chem. Soc., 2006, 128, 14278; M. Haruta and M. Daté, Appl. Catal. A, 2001, 222, 427.

10512 A. B. Laursen, K. T. Højholt, L. F. Lundegaard, S. B. Simonsen, S. Helveg, F. Schüth, M. Paul, J.-D. Grunwaldt, S. Kegnæs, C. H. Christensen and K. Egeblad, Angew. Chem. Int. Ed., 2010, 49, 3504; X. Zhang, X. Ke and H. Zhu, Chem. Eur. J., 2012, 18, 8048.

13 G. Lu, S. Li, Z. Guo, O. K. Farha, B. G. Hauser, X. Qi, Y. Wang, X. 110 Wang, S. Han, X. Liu, J. S. DuChene, H. Zhang, Q. Zhang, X. Chen, J. Ma, S. C. J. Loo, W. D. Wei, Y. Yang, J. T. Hupp and F. Huo, Nat. Chem., 2012, 4, 310; H. Liu, Y. Liu, Y. Li, Z. Tang and H. Jiang, J. Phys. Chem. C, 2010, 114, 13362; D. Esken, S. Turner, O. I. Lebedev, G. Van Tendeloo and R. A. Fischer, Chem. Mater., 2010, 22, 6393.

14 D. B. Akolekar and S. K. Bhargava, J. Mol. Catal. A, 2005, 236, 77; R. J. White, R. Luque, V. L. Budarin, J. H. Clark and D. J. Macquarrie, Chem. Soc. Rev., 2009, 38, 481.

15 H. Choi, S. R. Al-Abed, S. Agarwal and D. D. Dionysiou, Chem. 120 Mater., 2008, 20, 3649; A. Barau, V. Budarin, A. Caragheorgheopol, R. Luque, D. Macquarrie, A. Prelle, V. Teodorescu and M. Zaharescu, Catal. Lett., 2008, 124, 204.

16 M. Haruta, S. Tsubota, T. Kobayashi, T. Kageyama and M. J. Genet, J. Catal., 1993, 144, 175. 
17 N. Dimitratos, A. Villa, D. Wang, F. Porta, D. Su and L. Prati, $J$. Catal., 2006, 244, 113; F. Porta, L. Prati, M. Rossi and G. Scari, J. Catal., 2002, 211, 464.

18 A. Abad, A. Corma and H. García, Chem. Eur. J., 2008, 14, 212.

519 H. Guo, A. Al-Hunaiti, M. Kemell, S. Rautiainen, M. Leskela and T. Repo, ChemCatChem, 2011, 3, 1872.

20 Q. Huang, M. Ulutagay, P. A. Michener and S.-J. Hwu, J. Am. Chem. Soc., 1999, 121, 10323.

21 E. Williams, R. Leithall, R. Raja, M. T. Weller, Chem. Commun., $10 \quad 2013,49,249$.

22 R. Raja, S. Hermans, D. S. Shephard, B. F. G. Johnson, G. Sankar, S. Bromley and J. M. Thomas, Chem. Commun., 1999, 0, 1571; R. Raja, T. Khimyak, J. M. Thomas, S. Hermans and B. F. G. Johnson, Angew. Chem. Int. Ed., 2001, 40, 4638; R. D. Adams, E. M. Boswell,

15 B. Captain, A. B. Hungria, P. A. Midgley, R. Raja and J. M. Thomas, Angew. Chem. Int. Ed., 2007, 46, 8182; E. Gianotti, V. N. Shetti, M. Manzoli, J. A. L. Blaine, W. C. Pearl, R. D. Adams, S. Coluccia and R. Raja, Chem. Eur. J., 2010, 16, 820.

23 A. B. Hungria, R. Raja, R. D. Adams, B. Captain, J. M. Thomas, P.

20 A. Midgley, V. Golovko and B. F. G. Johnson, Angew. Chem. Int. Ed., 2006, 45, 4782.

24 R. Raja, R. D. Adams, D. A. Blom, W. C. Pearl, E. Gianotti and J. M. Thomas, Langmuir, 2009, 25, 7200.

25 S. R. Joshi, K. L. Kataria, S. B. Sawant and J. B. Joshi, Ind. Eng.

$25 \quad$ Chem. Res., 2004, 44, 325. 